\title{
NICE targeted screening to identify gestational diabetes: who are we missing?
}

\author{
CANEL KUCUK, ${ }^{1}$ ALI J CHAKERA, ${ }^{2}$ ELAINE MACANINCH ${ }^{3}$
}

\begin{abstract}
Background: Women with gestational diabetes (GDM) are at increased risk of perinatal complications. NICE currently recommends targeted screening of high-risk pregnant women. Our hospital's diagnostic pathway for GDM has changed from universal screening (glucose challenge test followed by oral glucose tolerance test) to NICE-recommended targeted screening.
\end{abstract}

Aims: We investigated whether women who would be missed by a targeted screening protocol had adverse clinical outcomes.

Methods: We reviewed the electronic records of all women with GDM who delivered between April and October 2016, during a period of universal screening. Treatment and birth outcomes were compared between those with NICE risk factors (RF) and those with no risk factors (NoRF). Differences were assessed using $X 2$ tests.

Results: 92 women with GDM gave birth during the six months studied. 32 (35\%) had no risk factors and would not currently be diagnosed with GDM. There was a significant difference in treatment between the NoRF and RF groups $(p=0.003)$. More women in the NoRF group were managed on diet and exercise alone ( $53 \%$ vs. $40 \%)$ and fewer NoRF women required insulin therapy $(19 \%$ vs. $45 \%)$. Delivery events were similar in the two groups $(p=0.23)$. Fewer women in the NoRF group had macrosomia (birth weight $>4000 \mathrm{~g}$ ) (NoRF $n=1(3.1 \%)$ vs. $R F \mathrm{n}=5$ $(8.3 \%))$. Admissions to the special care baby unit, predominantly for neonatal hypoglycaemia (capillary blood glucose $<2.2 \mathrm{mmol} / \mathrm{L}$ ), were greater in the NoRF group (NoRF $n=7(21.9 \%)$ vs. RF $n=5(8.3 \%))$. No stillbirths or shoulder dystocia occurred in either group.

Conclusions: Targeted screening for GDM using NICE guidelines would have missed over a third of women with GDM. Although women in the NoRF group required less

Brighton and Sussex Medical School, Brighton, UK

Department of Diabetes and Endocrinology, Royal Sussex County

Hospital, Brighton and Sussex University Hospitals, Brighton, UK

Department of Nutrition and Dietetics, Royal Sussex County Hospital,

Brighton and Sussex University Hospitals, Brighton, UK

Address for correspondence: Dr Canel Kucuk Brighton and Sussex Medical School, Southpoint building,

Royal Sussex County Hospital Brighton, BN2 5BE, UK

Email: canel.kucuk@nhs.net

https://doi.org/10.15277/bjd.2019.211 pharmacological treatment, they had adverse maternal and fetal outcomes equivalent to those of women with RF. Our results support universal screening of GDM.

Br J Diabetes 2019;19:25-28

Key words: gestational diabetes, screening, diagnosis, diabetes, glucose, hyperglycaemia

\section{Introduction}

Importance of diagnosing gestational diabetes

Women with gestational diabetes (GDM) are at increased risk of perinatal complications such as pre-eclampsia, shoulder dystocia and neonatal hypoglycaemia. ${ }^{1-3}$ Furthermore, these women face a long-term metabolic risk with a risk of up to $50 \%$ of developing diabetes mellitus in the first 5 years postpartum. ${ }^{4}$ The importance of identifying and managing women with GDM is well established.

Diagnosis of GDM: NICE versus WHO

There is currently no universal consensus for screening or diagnosis of GDM and guidance varies between authorities. The National Institute for Health and Care Excellence (NICE) currently recommends targeted screening of pregnant women using a 75 $\mathrm{g}$ oral glucose tolerance test (OGTT) at booking for women with previous GDM or at 24-28 weeks in the presence of the following risk factors: body mass index $(\mathrm{BMI})>30 \mathrm{~kg} / \mathrm{m}^{2}$, birth weight $>4.5 \mathrm{~kg}$, previous history of GDM in pregnancy, family history of diabetes, high-risk ethnic group. 5 GDM is diagnosed when fasting glucose is $\geq 5.6 \mathrm{mmol} / \mathrm{L}$ or 2 -hour post glucose $\geq 7.8 \mathrm{mmol} / \mathrm{L}$. $^{5}$ These diagnostic criteria are supported by a NICE cost-benefit analysis which concluded that the benefit of intervention may result in reduced risk associated with hyperglycaemia in pregnancy within the identified patient group. ${ }^{6}$

The World Health Organization (WHO) recommends universal testing for all pregnant women using a $75 \mathrm{~g}$ glucose diagnostic OGTT at 24-28 weeks. This diagnostic threshold (fasting $\geq 5.1 \mathrm{mmol} / \mathrm{L}$, 2 -hour $\geq 8.5 \mathrm{mmol} / \mathrm{L}$ ) is informed from data published by the Hyperglycaemia and Pregnancy Outcomes (HAPO) study which concluded that there is a linear relationship between hyperglycaemia and perinatal complications.7 Furthermore, internationally varied guidance for diagnosis of GDM exists. The Australian Diabetes in Pregnancy Society endorses the WHO one-step universal diagnostic OGTT testing. Similarly, the American Diabetes Association (ADA) supports a one-step OGTT diagnostic testing, although it recognises the American College of Obstetricians and Gynecologist's concerns that a one-step 
universal diagnostic process could lead to a steep rise in prevalence from $5-7 \%$ to $18 \%$ without significant improvement in clinical outcomes. Therefore, the ADA continues to advocate both a one-step OGTT and two-step glucose challenge test (GCT) followed by OGTT process. ${ }^{8}$

\section{Change in local diagnosis of GDM}

At our centre, up to 31 October 2016 we used a universal approach to diagnose GDM. At Brighton and Sussex University Hospital (BSUH) all pregnant women were screened with a blood glucose test 1 hour after a $50 \mathrm{~g} \mathrm{GCT}$. Those women with a 1 -hour value $\geq 7.8 \mathrm{mmol} / \mathrm{L}$ had a glucose tolerance test. Women with previous GDM or a $\mathrm{BMI} \geq 30 \mathrm{~kg} / \mathrm{m}^{2}$ were offered a glucose tolerance test at booking and at 24 and 28 weeks.

Since changing the protocol in line with the current NICE guidance, only women with risk factors are now screened for GDM at BSUH. ${ }^{5}$

\section{Study objective}

We wanted to assess treatment requirement and birth complications in women diagnosed with GDM at BSUH with NICE recognised risk factors versus those without, in order to identify the significance of missed opportunity for diagnosis under the NICE targeted screening protocol.

\section{Methods}

We reviewed the management of all singleton births between 1 April and 31 October 2016 to women diagnosed with GDM at Royal Sussex County Hospital (RSCH) before the protocol change.

Patients were separated into those with NICE recognised risk factors (RF) and those without (NoRF). We compared patient demographics, treatments and the mode of delivery between the two groups. We also compared birth weight and reviewed the incidence of birth complications, specifically shoulder dystocia, neonatal hypoglycaemia, special care baby unit (SCBU) admission or macrosomia (birth weight $>4 \mathrm{~kg}$ ).

Difference in birth weights was analysed using a t-test and categorical variables (mode of delivery and birth complications) were assessed using a $\times 2$ test.

\section{Results}

Of the 92 women with GDM identified during the study period, 60 had $\geq 1$ NICE recognised risk factors (RF group) and 32 had no risk factors (NoRF group).

The average age at delivery of patients in the RF and NoRF groups was 31.6 and 33.7 years, respectively $(p=0.07)$. Women in the NoRF group were by definition all white Caucasian with $\mathrm{BMl}<30 \mathrm{~kg} / \mathrm{m}^{2}$. The risk factors identified in the RF group are shown in Figure 1.

\section{Birth weight}

There was no difference in birth weight between the two groups (Figure 2A). The mean birth weight for births to RF patients was $3237 \mathrm{~g}$ (range 1457-4655 g) and the mean birth weight for
Figure 1. Number of women with individual risk factors (body mass index $(\mathrm{BMI})>30 \mathrm{~kg} / \mathrm{m} 2$, baby $>4.5 \mathrm{~kg}$, previous gestational diabetes (GDM), family history (FH) of diabetes, high-risk ethnicity) delivering at Royal Sussex County Hospital between 1 April and 31 October 2016. Note: data are greater than the total number of women with risk factors as individual women may have more than one risk factor

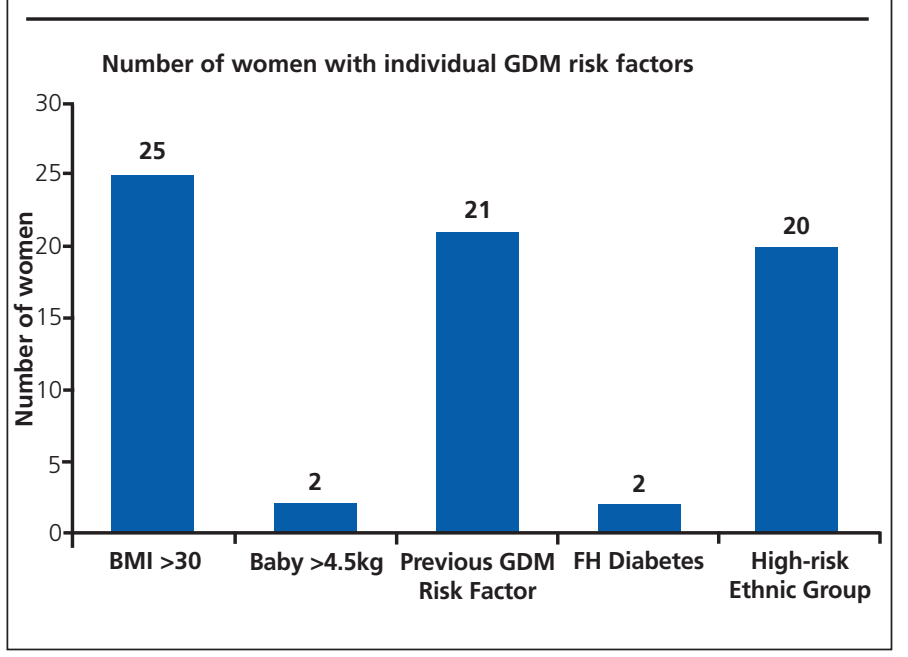

births to NoRF patients was $3363 \mathrm{~g}$ (range 2160-4245 g) $(p=0.12)$. Eight patients $(13.3 \%)$ in the RF group delivered prematurely ( $<37$ weeks gestation) compared with five patients $(15.6 \%)$ in the NoRF group. There was no difference in gestation at delivery $(p=0.24)$.

\section{Treatment}

A greater proportion of women in the RF group required some pharmacological treatment (metformin and/or insulin) compared with the NoRF group $(60.0 \%(n=32)$ vs. $46.9 \%(n=15)$ (Figure 2B). Twenty-four patients (40.0\%) in the RF group were managed on diet and exercise alone compared with 17 (53.1\%) in the NoRF group. Nine RF patients (15\%) were managed on metformin compared with nine $(28.1 \%)$ NoRF patients. Twentyseven patients in the RF group (45.0\%) required insulin compared with six patients (18.8\%) in the NoRF group.

\section{Mode of delivery}

There was no significant difference in mode of delivery (spontaneous vaginal delivery (SVD), assisted delivery, planned caesarean section and emergency caesarean section) in our study population ( $p=0.24$; Figure 2C). Thirty-seven patients in the RF group $(61.7 \%)$ delivered by SVD compared with $19(59.4 \%)$ in the NoRF group. Two RF patients (3.3\%) required assisted delivery (ventouse or forceps) compared with three NoRF patients (9.4\%). Thirteen patients in the RF group (21.7\%) delivered by planned caesarean section compared with seven $(21.9 \%)$ in the NoRF group. Seven RF patients (11.7\%) and three NoRF patients $(9.4 \%)$ delivered by emergency caesarean section. In summary: 
Figure 2. Comparison of outcomes between risk factor (RF) and no risk factor (NoRF) pregnancies. There was no difference in mean birth weight for births to RF patients (red) (3237 g, range 1457-4655 g) and births to NoRF patients (blue) (3363 g (range 2160-4245 g). More RF women (60\%) than NoRF women (47\%) needed pharmacological treatment. NoRF and RF women did not differ in mode of delivery.

(a) Birth weight

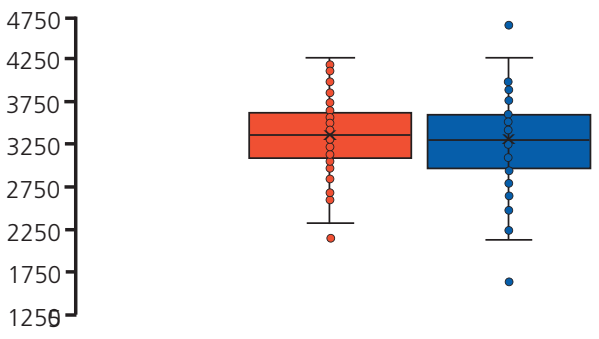

(c) Delivery events

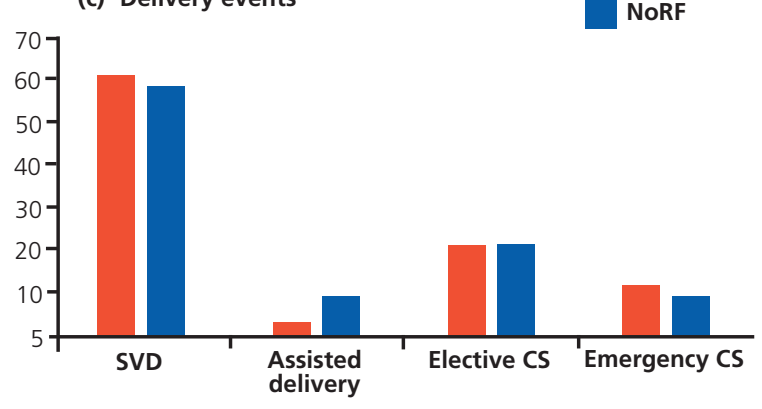

(b) Treatment

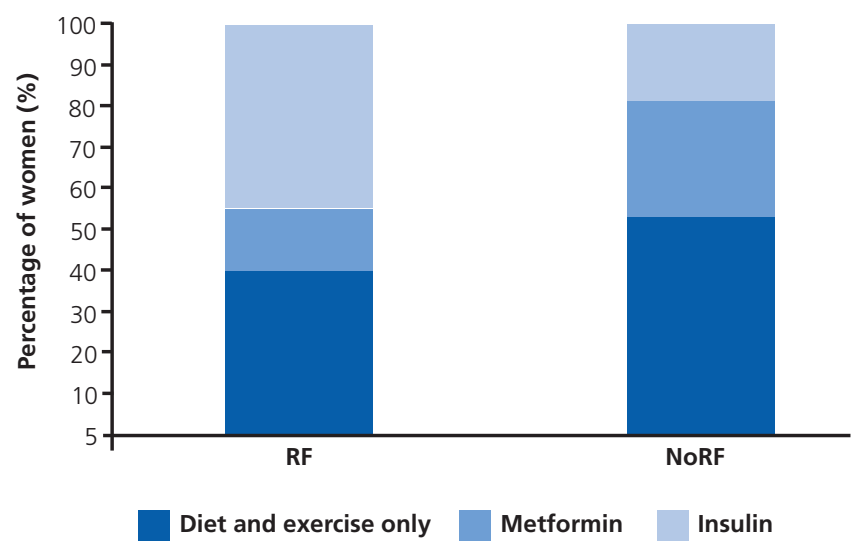

\section{Birth complications}

There were no cases of stillbirth or shoulder dystocia in the women over the measured period. Neonatal hypoglycaemic events were noted in $5.0 \%(n=3)$ of RF births and $12.5 \%(n=4)$ of NoRF births. SCBU admission was required for five RF births (8.3\%) and seven NoRF births (21.9\%). Furthermore, five RF births (8.3\%) and one NoRF birth (3.1\%) were macrosomic. We did not perform any statistical analysis on the incidence of birth complications as the number of total events was too low.

\section{Discussion}

\section{Main findings}

Our study indicates that targeted screening using NICE guidelines would have missed over a third of women with GDM. Fewer NoRF women required pharmacological intervention; however, these women had adverse maternal and fetal outcomes equivalent to those of women with RF.

\section{Strengths and limitations of the study}

The patients were separated into those with NICE recognised risk factors for GDM and those without. The patients were all treated within the same centre following their diagnosis. This enabled us to compare and contrast birth outcomes directly. However, this is a retrospective note review and thus our study is limited by the quality of note taking and assumption that all pregnant women were screened at $\mathrm{RSCH}$ during our study period. In addition, a larger population size would have enabled better analysis of correlative associations of significance. The lower than expected number of cases with a family history of diabetes may indicate poor quality of note taking where more than one risk factor is present.

Our conclusions about the impact of a diagnosis of GDM are limited by the absence of data relating to delivery outcomes within a negative comparator group over the study period. This limits discussion on cost effectivity of a GDM diagnosis.

\section{Interpretation of findings in relation to previously published work}

Universal diagnosis is not currently recommended by NICE guidance based on a cost-effectivity analysis which identified a significant cost-effectivity in terms of treatment of GDM in the risk factor group only. ${ }^{5}$ This was based on a theoretical cost effectivity analysis, which was derived from limited clinical data. ${ }^{6}$ Furthermore, the analysis did not take into account cost associated with long-term birth complications and macrosomia, rendering the analysis incomplete. ${ }^{9}$ The NICE cost-effectiveness analysis estimated that the extra cost of a universal GCT diagnostic method over targeted screening is marginal. However, this was only considering the cost of the test and did not take into account the cost of potential adverse outcomes. Our results show that there were adverse events which could influence the overall cost benefits. There is evidence to support universal OGTT screening for GDM as advised by WHO. Meek et al reported a prevalence of 


\section{Key messages}

- Targeted screening using NICE guidelines would have missed over a third of women with GDM

- These women had adverse maternal and fetal outcomes equivalent to those of women with RF

GDM of $6.42 \%$ diagnosed by universal screening and $4.12 \%$ using NICE criteria. In a retrospective review of 25,543 singleton births, they concluded that women may be at greater risk of maternal and infant complications where diagnosis is missed. ${ }^{10}$ A randomised controlled trial by Griffin et al demonstrated the benefit of universal screening, which increased uncomplicated vaginal deliveries and led to fewer complications. They concluded that universal screening facilitated earlier diagnosis and better glucose control, which may positively impact pregnancy outcomes. ${ }^{11}$ However, using data from a population of women taken from the HAPO and ATLANTA study, Jacklin et al concluded that screening-based diagnosis was more cost effective. ${ }^{12}$ Yet, a recent Cochrane review suggested that further research is required to conclude the cost effectiveness of a risk factor versus universal-based screening method. ${ }^{13}$ Furthermore, the NICE cost-benefit analysis demonstrates only a marginal increase in expense using universal screening and, although the analysis concludes that risk factor-based screening is cost effective, some complications demonstrated to occur in a patient with GDM have not been included within the NICE cost-benefit analysis, which may impact this assessment. ${ }^{14}$ In addition, a missed GDM diagnosis may prevent the chance to influence long-term maternal health and future risk of type 2 diabetes. The ATLANTIC DIP found that $28.4 \%$ of women with GDM go on to have diabetes or pre-diabetes in the first 1-5 years after pregnancy. ${ }^{15}$

\section{Implications for future research, policy and practice}

NICE guidance suggests that those women with GDM who are not identified by the NICE screening criteria have milder disease and thus a lower risk of complications. Our findings show that women without NICE risk factors still have significant complications and highlight the need for larger scale intervention studies.

\section{Conclusion}

Overall, our study suggests our current practice of diagnosis based on NICE guidance is missing women with GDM who may have preventable perinatal morbidity.

\section{Conflict of interest None.}

Funding None to declare.

Acknowledgements We thank Clare Gourlay, David Utting and the antenatal diabetes team at The Royal Sussex County Hospital for their support during data collection.

\section{References}

1. Barbour L, McCurdy C, Hernandez T, Kirwan J, Catalano P, Friedman J. Cellular mechanisms for insulin resistance in normal pregnancy and gestational diabetes. Diabetes Care 2007;30(Suppl 2):S112-S119. https://doi.org/ 10.2337/dc07-s202

2. Buckley B, Harreiter J, Damm P, et al. Gestational diabetes mellitus in Europe: prevalence, current screening practice and barriers to screening. A review. Diabet Med 2012;29:844-54. https://doi.org/10.1111/j.1464-5491. 2011.03541.x

3. Immanuel J, Simmons D. Screening and treatment for early-onset gestational diabetes mellitus: a systematic review and meta-analysis. Current Diabetes Rep 2017;17. https://doi.org/10.1007/s11892-017-0943-7

4. Venkataraman H, Sattar N, Saravanan P. Postnatal testing following gestational diabetes: time to replace the oral glucose tolerance test? Lancet Diabetes Endocrinol 2015;3:754-6. https://doi.org/10.1016/S22138587(15)00232-6

5. National Institute for Health and Care Excellence (NICE). Diabetes in pregnancy: management from preconception to the postnatal period. NICE guideline [NG3]. 2017. https://www.nice.org.uk/Guidance/NG3

6. National Institute for Health and Care Excellence (NICE). Costing statement: Diabetes in pregnancy. Implementing the NICE guideline on diabetes in pregnancy [NG3]. 2017. https://www.nice.org.uk/guidance/ng3/resources/ costing-statement- 3782989

7. Lowe L, Metzger B, Dyer A, et al. Hyperglycemia and Adverse Pregnancy Outcome (HAPO) study: associations of maternal A1C and glucose with pregnancy outcomes. Diabetes Care 2012;35:574-80. https://doi.org/ $10.2337 / \mathrm{dc} 11-1687$

8. Agarwal M. Gestational diabetes mellitus: an update on the current international diagnostic criteria. World J Diabetes 2015;6:782-91. https://doi.org/10.4239/wjd.v6.i6.782

9. Meek C. Natural selection? The evolution of diagnostic criteria for gestational diabetes. Ann Clin Biochem 2016;54:33-42. https://doi.org/ 10.1177/0004563216674743

10. Meek C, Lewis H, Patient C, Murphy H, Simmons D. Diagnosis of gestational diabetes mellitus: falling through the net. Diabetologia 2015;58:2003-12. https://doi.org/10.1007/s00125-015-3647-z

11. Griffin $M$, Coffey $M$, Johnson $H$, et al. Universal vs. risk factor-based screening for gestational diabetes mellitus: detection rates, gestation at diagnosis and outcome. Diabet Med 2000;17:26-32. https://doi.org/10.1046/.14645491.2000.00214.x

12. Jacklin $P$, Maresh M, Patterson $C$, et al. A cost-effectiveness comparison of the NICE 2015 and WHO 2013 diagnostic criteria for women with gestational diabetes with and without risk factors. BMJ Open 2017;7(8): e016621. https://doi.org/10.1136/bmjopen-2017-01662

13. Tieu J, McPhee A, Crowther C, Middleton P, Shepherd E. Screening for gestational diabetes mellitus based on different risk profiles and settings for improving maternal and infant health. Cochrane Database of Systematic Reviews [online]. August 2017. https://www.cochranelibrary.com/cdsr/ doi/10.1002/14651858.CD007222.pub4/full

14. National Institute for Health and Care Excellence (NICE). Diabetes in pregnancy. Management of diabetes and its complications from preconception to the postnatal period. NICE Guideline 3. Methods, evidence and recommendations. 2015. https://www.nice.org.uk/guidance/ng3/evidence/fullguideline-3784285

15. Crowe C, Noctor E, Carmody LA, et al. ATLANTIC DIP: The prevalence of pre-diabetes/type 2 diabetes in an Irish population with gestational diabetes mellitus 1-5 years post index pregnancy. BMC Proc 2012;6(Suppl 4):035. https://doi.org/10.1186/1753-6561-6-S4-035 